\title{
EQUILIBRIUM PROBLEM IN A ROTATING CONVECTION ZONE
}

\author{
YU. V. VANDAKUROV \\ Physical and Technical Institute A. F. Ioffe, Leningrad, U.S.S.R.
}

\begin{abstract}
Taking into account effects produced by the convective motions, the equilibrium problem for a rotating star becomes greatly complicated. We consider this problem for the case of slow rotation in the following approximations.

We treat the convection zone as a medium with turbulent viscosity and turbulent thermal conductivity. However, we take into account the nonlinear effects produced by the most rapidly growing perturbations. The corresponding nonlinear terms are calculated by using the solution of linear perturbed equations. Each independent convective mode is supposed to have initially the same amount of kinetic energy.

In the limit of small turbulent viscosity, we show that unstable convective perturbations produce a mean azimuthal force due to which rigid rotation appears not to be in the equilibrium. For the case of small-scale perturbations and latitudinal differential rotation, this force is analogous to the viscous force, but the coefficient of viscosity is negative.

We suggest that such a force maintains the differential rotation of the solar convection zone. Note that in the case under consideration the latitudinal dependence of the solar heat flux is small. However, difficulties arise due to different conditions at different depths in the convection zone. In this connection, a hypothesis is put forward that magnetic fields are also necessary to get balance in full. A model of the solar cycle is discussed which is similar in some respects to the well-known Babcock model. We propose, however, that the field reversal takes place in the lower layers of that zone where fields are intensified.
\end{abstract}

\section{DISCUSSION}

Durney: You consider the modes with the largest growth rate which I will designate by $M$. The influence of rotation on a mode depends on its strength and dimensions. Could not this influence be larger for modes with smaller growth rates but larger dimensions than $M$ ?

Vandakurov: The horizontal mode number $L$ in the final formula for the azimuthal force refers to some dominant mode. It seems likely that this mode corresponds to the mode having the faster growth rate (of course, this rate should be found from nonlinear perturbed equations). If some other value of $L$ is more suitable, it can be inserted in the atove formula. To study the problem more exactly an extension of the calculations to cover different values of $L$ is needed.

Gilman: Your theory requires a magnetic field, which, in turn would have cyclic variations with time. By what fraction would your differential rotation change with time to feed back from the magnetic field?

Vandakurov: The problem of time variations of differential rotation has not been considered in detail. We think that our model can be put in accordance with the well-known models by Babcock and Leighton (with the modifications concerning the field-reversal mechanism and the basic justification of the existence of the solar activity cycle). Thus, the explanation of the main features of the cycle seems to be similar to that in the above mentioned models. But indeed, the time variations of rotation velocity should be present in our model. In some layers field amplification is smaller or larger than that required by the equilibrium condition, thus, there, redistribution of angular velocity occurs. The rotation velocity is kept constant only in some averaged value. 